\title{
Curcumin ameliorates dopaminergic neuronal oxidative damage via activation of the Akt/Nrf2 pathway
}

\author{
QUNLI CUI ${ }^{1}, \mathrm{XIN} \mathrm{LI}^{1}$ and HONGCAN ZHU ${ }^{2}$ \\ ${ }^{1}$ Department of Neurology, The Second Teaching Hospital of Zhengzhou University, Zhengzhou, Henan 450014; \\ ${ }^{2}$ Department of Neurology, The First Affiliated Hospital of Zhengzhou University, Zhengzhou, Henan 450052, P.R. China
}

Received January 19, 2015; Accepted November 6, 2015

DOI: $10.3892 / \mathrm{mmr} .2015 .4657$

\begin{abstract}
Parkinson's disease (PD) is an age-related complex neurodegenerative disease that affects $\leq 80 \%$ of dopaminergic neurons in the substantia nigra pars compacta ( $\mathrm{SNpc})$. It has previously been suggested that mitochondrial dysfunction, oxidative stress and oxidative damage underlie the pathogenesis of PD. Curcumin, which is a major active polyphenol component extracted from the rhizomes of Curcuma longa (Zingiberaceae), has been reported to exert neuroprotective effects on an experimental model of PD. The present study conducted a series of in vivo experiments, in order to investigate the effects of curcumin on behavioral deficits, oxidative damage and related mechanisms. The results demonstrated that curcumin was able to significantly alleviate motor dysfunction and increase suppressed tyrosine hydroxylase (TH) activity in the SNpc of rotenone (ROT)-injured rats. Biochemical measurements indicated that rats pretreated with curcumin exhibited increased glutathione (GSH) levels, and reduced reactive oxygen species activity and malondialdehyde content. Mechanistic studies demonstrated that curcumin significantly restored the expression levels of heme oxygenase-1 and NAD(P)H:quinone oxidoreductase 1, thus ameliorating ROT-induced damage in vivo, via the phosphorylation of Akt and nuclear factor erythroid 2-related factor 2 (Nrf2). Further studies indicated that the Akt/Nrf2 signaling pathway was associated with the protective role of curcumin
\end{abstract}

Correspondence to: Dr Qunli Cui, Department of Neurology, The Second Teaching Hospital of Zhengzhou University, 2 Jingba Road, Zhengzhou, Henan 450014, P.R. China

E-mail: qunlicui@163.com

Abbreviations: PD, Parkinson's disease; SNpc, substantia nigra pars compacta; Nrf2, nuclear factor erythroid 2-related factor 2; $\mathrm{PI} 3 \mathrm{~K}$, phosphoinositide 3-kinase; NQO1, NAD(P)H:quinone oxidoreductase 1; LPO, lipid peroxidation; MDA, malondialdehyde; ROS, reactive oxygen species; HO-1, heme oxygenase-1; ROT, rotenone; TH, tyrosine hydroxylase; GSH, glutathione; shRNA, short hairpin RNA

Key words: Parkinson's disease, rotenone, curcumin, oxidative damage, dopaminergic neurons in ROT-treated rats. Inhibiting the Akt/Nrf2 pathway using a lentiviral vector containing Nrf2-specific short hairpin RNA, or the phosphoinositide 3-kinase inhibitor LY294002, markedly reduced the expression levels of TH and GSH, ultimately attenuating the neuroprotective effects of curcumin against oxidative damage. These results indicated that curcumin was able to significantly ameliorate ROT-induced dopaminergic neuronal oxidative damage in the $\mathrm{SNpc}$ of rats via activation of the Akt/Nrf2 signaling pathway.

\section{Introduction}

Parkinson's disease (PD) is the second most common progressive neurodegenerative disorder worldwide, which is well characterized by the degeneration of dopaminergic neurons in the substantia nigra pars compacta (SNpc). The diagnosis of $\mathrm{PD}$ is associated with various symptoms of motor dysfunction, including progressive resting tremor, rigidity, bradykinesia and postural instability (1). It has previously been suggested that the progression of PD may be associated with mitochondrial dysfunction (2), and mitochondrial complex I inhibition is not limited to the brain in PD. The brain is particularly susceptible to oxidative damage due to its high expression of polyunsaturated fatty acids and relatively low antioxidant activity (3); oxidative stress has been reported to have a crucial role in the pathogenesis of $\mathrm{PD}$, and is considered the most important underlying mechanism (4). In addition, oxidative stress and mitochondrial dysfunction have been identified as early events during the process of neurodegeneration (5). The majority of research regarding neurodegeneration in PD has focused on the brain, and most of the resultant oxidative damage has been detected in the SNpc. A marked decrease in glutathione (GSH) levels in the SNpc is considered to be the earliest triggering event of neurodegeneration, which precedes oxidative stress (6), mitochondrial dysfunction and dopamine loss during early PD (7). Therefore, restoration of cellular GSH levels may prevent the initiation of PD.

Rotenone (ROT) is an active herbicide that can be used to induce dopaminergic neuronal oxidative damage in experimental animal models of PD (8). The polyphenolic flavonoid curcumin [1,7-bis(4-hydroxy-3-methoxyphenyl)-1,6-heptadiene-3,5-dione], which is a principle coloring agent extracted from the rhizomes of Curcuma longa (9), is a dietary spice that is often used in Indian cuisine and medicine (10). Previous studies have demonstrated that curcumin possesses various 
properties, including antioxidant (11), anti-inflammatory (12), anticancer (13), iron-chelating (14) and neuroprotective activities (15). Notably, previous research regarding various experimental models of PD strongly supports the clinical application of curcumin in PD (16). The specific underlying mechanism is not fully understood; however, curcumin has exerted promising antioxidant effects in various cell and animal models of PD through modulating the generation of GSH, reactive oxygen species (ROS) and malondialdehyde (MDA) $(9,17,18)$. Furthermore, our previous study demonstrated that curcumin was able to attenuate ROT-induced cytotoxicity by exerting antioxidative and antiapoptotic activity in PC12 rat pheochromocytoma cells, thus suggesting that curcumin has neuroprotective effects on the dopaminergic neurons of PD (19). However, there is still a lack of data with regards to the molecular mechanism underlying the protective effects of curcumin in animal models of neurodegenerative diseases.

Previous studies have demonstrated that Akt phosphorylation facilitates nuclear factor erythroid 2-related factor 2 (Nrf2) translocation into the nucleus, which has an important role in the defense against oxidative stress by regulating the expression levels of antioxidant enzymes, including heme oxygenase (HO)-1 and $\mathrm{NAD}(\mathrm{P}) \mathrm{H}$ :quinone oxidoreductase 1 (NQO1) (20-22). However, the majority of studies regarding the effects of ROT or curcumin on the progression of PD have focused on in vitro cell models $(18,19,23)$. The present study aimed to investigate the effects of curcumin on ROT-induced neurotoxicity and oxidative dysfunction, and to determine the underlying mechanism associated with PD progression.

\section{Materials and methods}

Animal care and cell culture. A total of 50 male Lewis rats (age, 7-8 weeks; weight, 260-280 g) were purchased from Beijing Vital River Laboratory Animal Technology Co., Ltd. (Beijing, China). The rats were housed in a room maintained at $22^{\circ} \mathrm{C}$ with $40-70 \%$ humidity, under a 12 -h light/dark cycle with ad libitum access to drinking water and a standard pellet diet. All scientific procedures were carried out in accordance with the relevant Guidelines in Neuroscience Research and the Care and Use of Laboratory Animals of the Second Affiliated Hospital of Zhengzhou University (Zhengzhou, China). The present study was approved by the local ethics committee of Zhengzhou University (Zhengzhou, China).

Human embryonic kidney (HEK)293T cells and SK-N-SH human neuroblastoma cells were obtained from the American Type Culture Collection (Manassas, VA, USA). The cells were cultured in Dulbecco's modified Eagle's medium (Gibco; Thermo Fisher Scientific, Inc., Waltham, MA, USA) supplemented with $4.5 \mathrm{~g} / 1$ high glucose, $2 \mathrm{mM}$ L-glutamine, $1 \mathrm{mM}$ sodium pyruvate (Thermo Fisher Scientific, Inc.) and $10 \%$ heat-inactivated fetal bovine serum (Gibco; Thermo Fisher Scientific, Inc.) at $37^{\circ} \mathrm{C}$ in an atmosphere containing $5 \% \mathrm{CO}_{2}$ and $95 \%$ air.

Experimental design. Rotenone (Sigma-Aldrich, St. Louis, MO, USA) was dissolved in sunflower seed oil at $1.5 \mathrm{mg} / \mathrm{ml}$. The rats were divided randomly into four groups ( $\mathrm{n}=6 /$ group): (i) The CON group, in which rats were injected with sunflower seed oil ( $0.5 \mathrm{ml} / \mathrm{kg} /$ day, twice daily) as a vehicle for 50 days; (ii) the ROT + curcumin treatment group (Sigma-Aldrich), in which rats were intragastrically administered curcumin (100 mg/kg) twice a day for 50 days, and ROT was subcutaneously administered twice a day at $1 \mathrm{ml} / \mathrm{kg} /$ day on day 4 of curcumin treatment; (iii) the ROT treatment group, in which the rats received the same as the ROT + curcumin group beginning on day 1, except that gum arabica (Sigma-Aldrich) was administered instead of curcumin; (iv) the curcumin treatment group, in which the rats received the same as the ROT + curcumin group, except that sunflower seed oil was administered instead of ROT. During the experimental period, the food intake was monitored daily and body weight was measured regularly. Behavioral assessments were carried out among rats of all groups. Subsequently, the rats were sacrificed by decapitation, and tissues were removed from the brain on a Petri dish placed on ice under a microscope (Olympus BX50, Tokyo, Japan). The SNpc regions were stored at $-80^{\circ} \mathrm{C}$, and the cytosolic fraction was subsequently extracted and subjected to various biochemical investigations and western blot analyses.

Apomorphine-induced rotations. A total of $24 \mathrm{~h}$ after the final injection, the rotational behavior of the rats was determined following intraperitoneal injection with freshly prepared apomorphine hydrochloride (1 mg/kg, s.c.; Sigma-Aldrich) for 5-10 min. Subsequently, full $360^{\circ}$ unilateral turns were recorded in a $10 \mathrm{~min}$ period. The rats that turned unilaterally $>7$ cycles/min, were considered successful models of PD.

Catalepsy and open field tests. In the catalepsy test, rats were hung by the paws on a vertical grid $(25 \times 45 \mathrm{~cm}, 1 \mathrm{~cm}$ space between each wire). The time taken for the rats to move their paws or to perform any sort of initial movement was noted. The rats were then placed with both forepaws on a bar $10 \mathrm{~cm}$ above, parallel from the base in a half-rearing position. The paw removal latency was noted, and the maximum descent latency was fixed at $180 \mathrm{sec}$ for both tests.

Behavioral tests were carried out in an open field using a spontaneous activity instrument (Shanghai Jiliang Software Technology Co., Ltd., Shanghai, China), including four boxes $(40 \times 40 \times 50 \mathrm{~cm})$. Each box housed one rat at a time. An infrared camera was attached to the top of the box to record the movement time, movement distance and movement speed for $1 \mathrm{~h}$. Subsequently, the video images were sent to a computer and analyzed with the JLBehv-LAR-4 software (Shanghai Jiliang Software Technology Co., Shanghai, China).

Biochemical measurements of GSH, ROS and MDA. The obtained SNpc regions were immediately homogenized using an ultrasonic cell disruptor (Model $150 \mathrm{~V} / \mathrm{T}$; BioLogics, Manassas, VA, USA), and then subjected to centrifugation at $14,000 \mathrm{x} \mathrm{g}$ for $15 \mathrm{~min}$ at $4^{\circ} \mathrm{C}$. The supernatant was used for the determination of the activity of various enzyme activities. GSH content in the SNpc regions was measured as described previously (24), and the fluorescence was measured using a spectrofluorometer (Model RF-5000, Shimadzu Corporation, Tokyo, Japan). GSH concentration was calculated from a standard curve and expressed as $\mu \mathrm{g} \mathrm{GSH} / \mathrm{mg}$ protein. ROS generation in the $\mathrm{SNpc}$ regions was assayed using dihydrodichlorofluorescein diacetate, as described previously (25). ROS formation was 
quantified from a dichlorodihydrofluorescein (DCF) standard curve and expressed as pmol DCF formed $/ \mathrm{min} / \mathrm{mg}$ protein. Lipid peroxidation (LPO) levels in the cytosol of the SNpc were quantified by measuring the formation of thiobarbituric acid reactive substances (TBARS), as described previously (26). The absorbance of TBARS adducts was measured at $532 \mathrm{~nm}$, which was used to quantify MDA levels. The commercial kits used to measure the levels of GSH, ROS and MDA were purchased from Nanjing Jiancheng Bioengineering Institute (Nanjing, China) according to the manufacturer's instructions.

Lentivirus preparation. HEK293T cells were co-transfected with envelope plasmid, packaging plasmid and expression plasmid (pLKO.1; Sigma-Aldrich) containing the shRNA using Lipofectamine ${ }^{\circledR} 2000$ (Invitrogen; Thermo Fisher Scientific, Inc.) and the Lentiviral Packaging Mix (Sigma-Aldrich), according to the manufacturers' protocols. The short hairpin (sh)RNA sequence targeting coding regions of the rat Nrf2 gene (shNrf2) and the control shScramble plasmid (GenePharma Co., Ltd., Shanghai, China) were used. The cell supernatant containing the lentivirus was collected, and filtered through membrane filters $(0.45-\mu \mathrm{m}$ pore size, Millipore Corporation, Bedford, MA, USA). Then, viral supernatant was concentrated by ultracentrifugation for $2 \mathrm{~h}$ at $50,000 \mathrm{x} \mathrm{g}$ and $4^{\circ} \mathrm{C}$ and stored at $-80^{\circ} \mathrm{C}$ until further use. The number of relative infection units (RIU) was determined by infecting SK-N-SH cells with a serial dilution.

Infusion with a shNrf2 lentivirus or phosphoinositide 3-kinase (PI3K) inhibitor LY294002. In the shNrf2 lentivirus experiment, nine groups ( $\mathrm{n}=6 /$ group) were designed: $\mathrm{CON}$ group; shScramble (control) group; shScramble + ROT group; shScramble + curcumin group; shScramble + ROT + curcumin group; shNrf2 group; shNrf2 + ROT group; shNrf2 + curcumin group; shNrf2 + ROT + curcumin group. Lentiviruses containing shScramble or shNrf2 $\left(2 \times 10^{7}\right.$ RIU; $\left.10 \mu \mathrm{l}\right)$ were infused into the $\mathrm{SNpc}$ regions with an infusion rate of $0.5 \mu \mathrm{l} / \mathrm{min}$. After $48 \mathrm{~h}$ recovery, the rats were treated with ROT and/or curcumin for 50 days as described previously.

In the LY294002 experiment, five groups (n=6/group) were designed: CON group; LY294002 (control) group; LY294002 + ROT group; LY294002 + curcumin group; LY294002 + ROT + curcumin group. ALZET ${ }^{\circledR}$ osmotic minipumps $(0.5 \mu \mathrm{l} / \mathrm{h}$; model 1007D; DURECT Corporation, ALZET Osmotic Pumps, Cupertino, CA, USA) were subcutaneously implanted on the back of the rats over the latissimus dorsi muscles, and were connected by catheters to the infusion cannula for intracerebroventricular infusion. The inhibitor-treated rats received osmotic minipumps filled with artificial cerebral spinal fluid (aCSF; Gibco; Thermo Fisher Scientific, Inc.) alongside $0.1 \mu \mathrm{g} / \mu \mathrm{l} \mathrm{LY} 294002$ (Sigma-Aldrich). The CON rats received equal volumes of phosphate-buffered saline in aCSF. The pumps were implanted 5 min after the first injection of ROT or curcumin, and remained active for 7 days. The rats were treated with ROT and curcumin for 50 days, as described previously. Subsequently, all rats were sacrificed and the SNpc regions of the brains were collected for western blot analysis.

Western blot analysis. Tissues were homogenized on ice using radioimmunoprecipitation assay lysis buffer (Beyotime
Institute of Biotechnology, Shanghai, China) and were centrifuged at $14,000 \times \mathrm{g}$ for $15 \mathrm{~min}$ at $4^{\circ} \mathrm{C}$. Protein concentration was determined using the Pierce Bicinchoninic Acid Protein Assay kit (Pierce Biotechnology, Inc., Rockford, IL, USA). Equal amounts of protein (25 $\mu \mathrm{g}$ per lane) were loaded onto $10 \%$ sodium dodecyl sulfate-polyacrylamide gel (Shanghai Sangon Biotechnology Co. Ltd., Shanghai, China), prior to being transferred onto polyvinylidene difluoride membranes (PVDF; Millipore Corporation). Subsequently, the membranes were blocked with $5 \%$ non-fat milk in $0.1 \%$ Tris-buffered saline with Tween-20 (TBST; Shanghai Sangon Biotechnology Co. Ltd.) for $1 \mathrm{~h}$ at room temperature, followed by incubation with the primary antibodies overnight at $4{ }^{\circ} \mathrm{C}$. The primary antibodies used were rabbit anti-tyrosine hydroxylase (TH) polyclonal antibody (cat. no. T8700; Sigma-Aldrich), mouse anti- $\beta$-actin monoclonal antibody (cat. no. A1978) and rabbit anti-HO-1 polyclonal antibody (cat. no. H4535) (dilution, 1:1,000; Sigma-Aldrich); rabbit anti-NQO1 polyclonal antibody (cat. no. sc-25591) and rabbit anti-Nrf2 polyclonal antibody (cat. no. sc-722) (dilution, 1:1,000; Santa Cruz Biotechnology, Inc., Dallas, TX, USA); rabbit anti-phosphorylated (p)-Nrf2 (Ser40) monoclonal antibody (cat. no. ab76026, dilution, 1:5,000; Abcam, Cambridge, MA, USA); rabbit anti-Akt polyclonal antibody (cat. no. \#9272) and rabbit anti-p-Akt (Ser473) polyclonal antibody (cat. no. \#9271) (dilution, 1:1,000; Cell Signaling Technology, Inc., Beverly, MA, USA). After incubation with appropriate goat anti-rabbit or anti-mouse immunoglobulin $\mathrm{G}$ conjugated to horseradish peroxidase (dilution, 1:5,000; Beyotime Institute of Biotechnology) for $1 \mathrm{~h}$ at room temperature, the resultant protein bands were visualized by enhanced chemiluminescence (Beyotime Institute of Biotechnology). The absorbance values of target proteins were determined using Gel-Pro Analyzer version 4.0 software (Media Cybernetics, Inc., Rockville, MD, USA).

Statistical analysis. Data are presented as the mean \pm standard deviation representative of three individual experiments performed in triplicate. Statistical analyses were conducted using one-way analysis of variance followed by the Student Newman-Keuls test. Statistical analyses were performed using GraphPad Prism software 5.0 (GraphPad Software Inc., La Jolla, CA, USA). P<0.05 was considered to indicate a statistically significant difference.

\section{Results}

Curcumin partly alleviates ROT-induced symptoms of PD. Almost all ROT-treated rats displayed significant weight loss during the first 10 days of ROT treatment, which was reversed after curcumin pretreatment. In the CON and curcumin-treated rats, body weight increased slowly and no significant difference was observed (Fig. 1A). In the rotational behavior test, the curcumin-treated rats exhibited similar numbers of rotation $(5.1 \pm 0.26$ turns $/ 10 \mathrm{~min})$, as compared with the CON group (5.6 \pm 0.24 turns/10 min; $\mathrm{P}>0.05)$; however, the ROT-treated rats exhibited markedly higher rotational numbers $(130 \pm 20.16$ turns/10 min; $\mathrm{P}<0.05)$. Notably, curcumin administration markedly suppressed the ROT-induced enhanced rotational behavior $(52 \pm 8.8$ turns/10 min; $\mathrm{P}<0.05$ ) (Fig. 1B). 


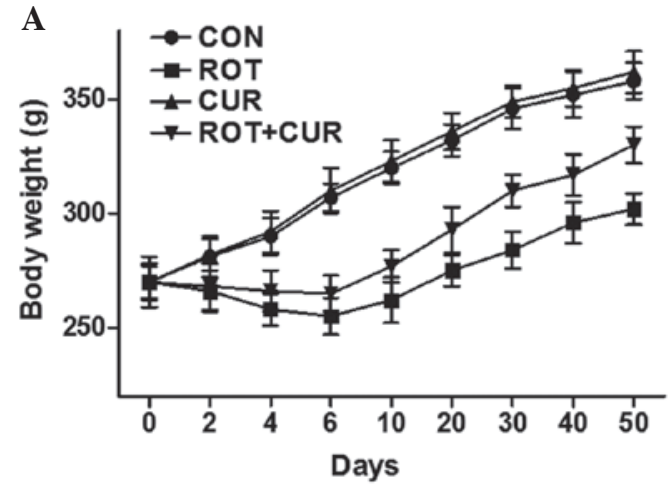

C

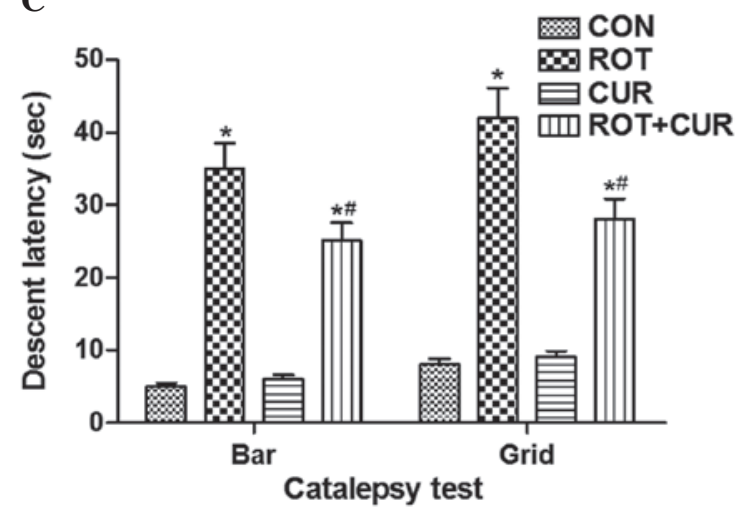

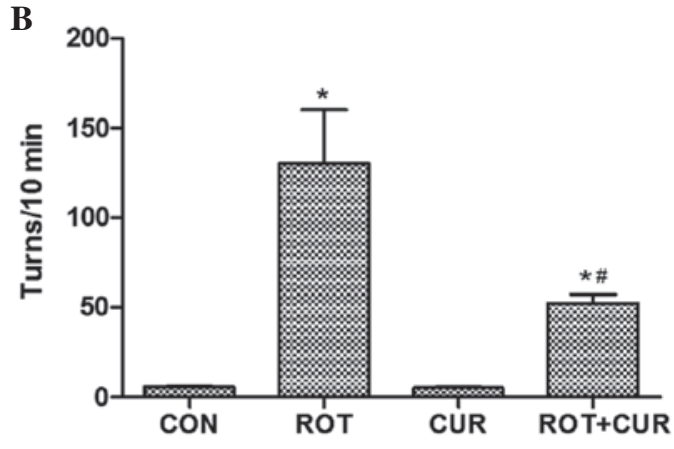

D

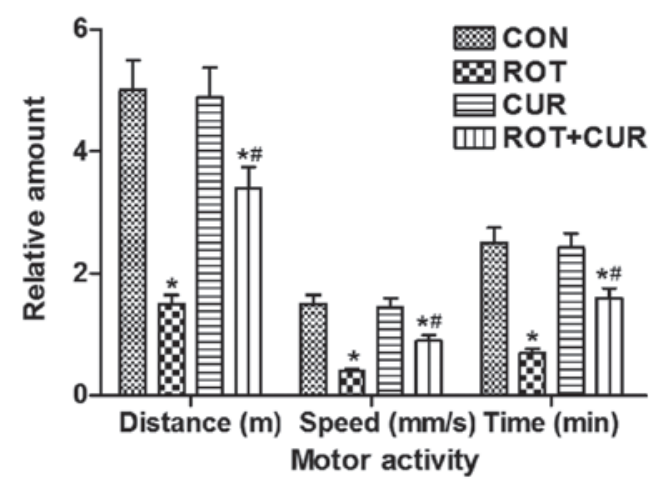

Figure 1. Effects of curcumin (CUR) and rotenone (ROT) on parkinsonian symptoms in rats. (A) Body weight of the rats was recorded daily and no mortality occurred. (B) Rotational behavior of all rats was recorded after 50 days of treatment with ROT and CUR. (C) Catalepsy of the rats was measured on a vertical grid and horizontal bar. (D) Locomotor activity assay of the rats recorded the movement time (min), movement distance (m) and movement speed (mm/s). Data are presented as the mean \pm standard deviation. ${ }^{\text {}} \mathrm{P}<0.05$ vs. the $\mathrm{CON}$ group. ${ }^{"} \mathrm{P}<0.05$ vs. the ROT group $(\mathrm{n}=6)$.

ROT-treated rats developed typical disordered behavioral features, including catalepsy, unsteady movement and hunched posture to varying degrees. ROT-treated rats exhibited prolonged descent latency in the grid and bar test (Fig. 1C) and were considered hypokinetic; the movement time, movement distance and movement speed of the ROT-treated rats were markedly reduced, as compared with the CON group (Fig. 1D). Furthermore, curcumin pretreatment alleviated these symptoms, and no obvious PD-associated postural or behavioral features were observed in the CON and curcumin-treated groups. These data suggest that PD may be induced in rats via chronic treatment with ROT, and the parkinsonian symptoms may be partly alleviated following curcumin pretreatment.

Curcumin ameliorates ROT-induced dopaminergic neuronal damage and oxidative injury. The present study aimed to determine the protective effects of curcumin on the neurochemical features of dopaminergic neurons in ROT-treated rats. Western blot analysis revealed that curcumin pretreatment restored the ROT-induced loss of TH protein, which is considered a marker of dopaminergic neurons, in the $\mathrm{SNpc}$ (Fig. 2A). ROS generation in the SNpc was significantly enhanced $(\sim 105 \%)$ following treatment with ROT exposure, as compared with in the CON group (Fig. 2B). Similarly, ROT-treated rats displayed higher levels of LPO (MDA levels) with a $43 \%$ elevation, as compared with the CON rats (Fig. 2C). Furthermore, GSH content was reduced following treatment with ROT with a $32 \%$ reduction, as compared with the CON group (Fig. 2D). Conversely, treatment with curcumin resulted in an increase in GSH levels, and a decrease in the accumulation of ROS and MDA levels induced by ROT treatment. These results suggest that ROT-induced oxidative stress may exert significant damage on dopaminergic neurons in the SNpc, and curcumin pretreatment may be partially neuroprotective against ROT exposure.

Curcumin activates the Akt/Nrf2-regulated antioxidant system. The protective effects of Akt/Nrf2 signaling against oxidative stress have previously been reported in PD (26); therefore, the present study aimed to explore whether the Akt/Nrf2 antioxidant system was associated with the protective effects of curcumin in ROT-treated rats. Curcumin pretreatment prevented the ROT-induced reductions of HO-1 and NQO1 protein expression, and Akt/Nrf2 phosphorylation (Fig. 3A and B). Collectively, these data suggest that curcumin may exhibit antioxidant and neuroprotective effects via activation of the Akt/Nrf2 signaling pathway in the SNpc dopaminergic neurons of ROT-induced parkinsonian rats.

shNrf2 lentivirus and LY294002 suppress curcumin-mediated neuroprotection. Transfection with a shNrf2 lentivirus or sublethal chronic exposure to LY294002 prior to treatment with ROT/curcumin, resulted in a failure of curcumin to prevent ROT-induced reductions in TH and GSH expression (Fig. 4A and B). In addition, Nrf2 phosphorylation was 
A

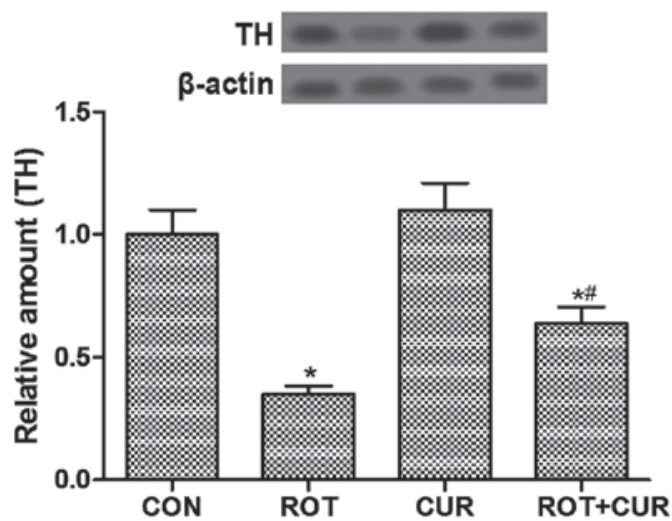

C

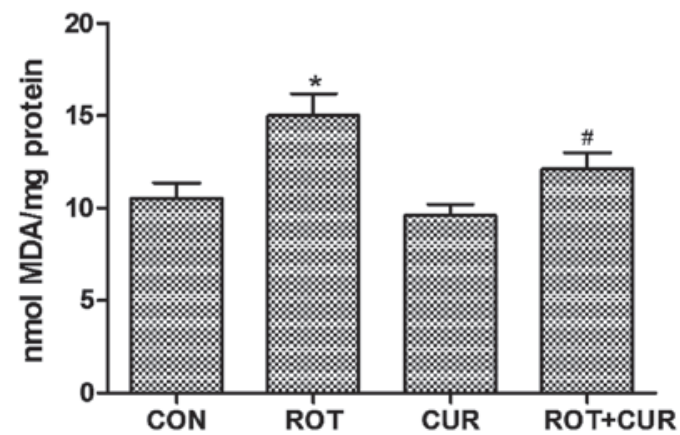

B

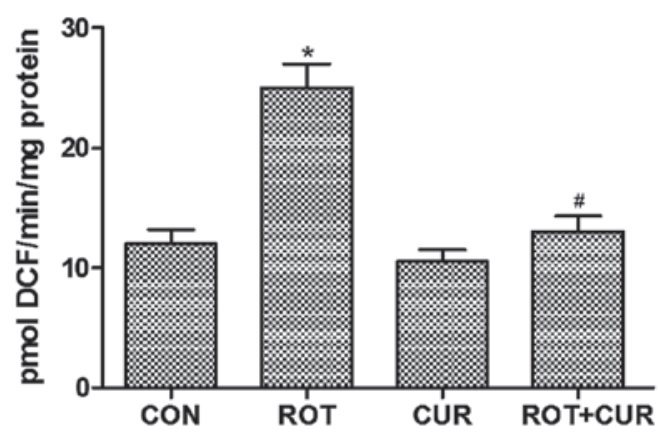

D

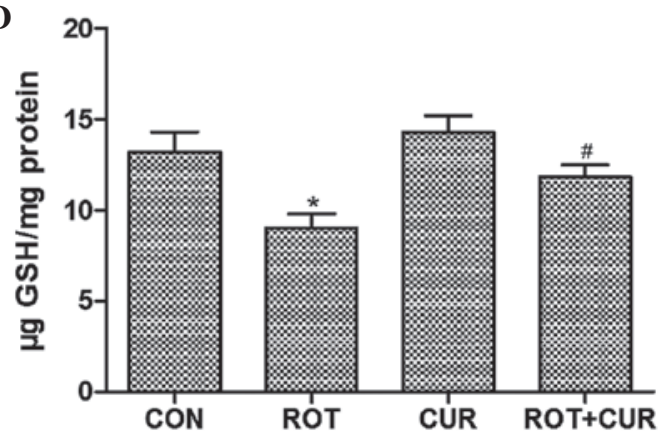

Figure 2. Effects of curcumin (CUR) and rotenone (ROT) on the state of dopaminergic neurons, and the levels of reactive oxygen species (ROS), malondialdehyde (MDA) and glutathione (GSH) in the substantia nigra pars compacta ( $\mathrm{SNpc}$ ) of rats. (A) SNpc sections were lysed and subjected to western blotting with anti-tyrosine hydroxylase (TH) antibody. Quantification analysis was performed using Gel-Pro Analyzer 4.0 and normalized to $\beta$-actin. Markers of oxidative status: (B) ROS, (C) MDA and (D) GSH, were examined using commercial kits. Data are presented as the mean \pm standard deviation. "P< $<0.05$ vs. the CON group. ${ }^{\text {}} \mathrm{P}<0.05$ vs. the ROT group ( $\left.\mathrm{n}=6\right)$. $\mathrm{DCF}$, dichlorodihydrofluorescein.
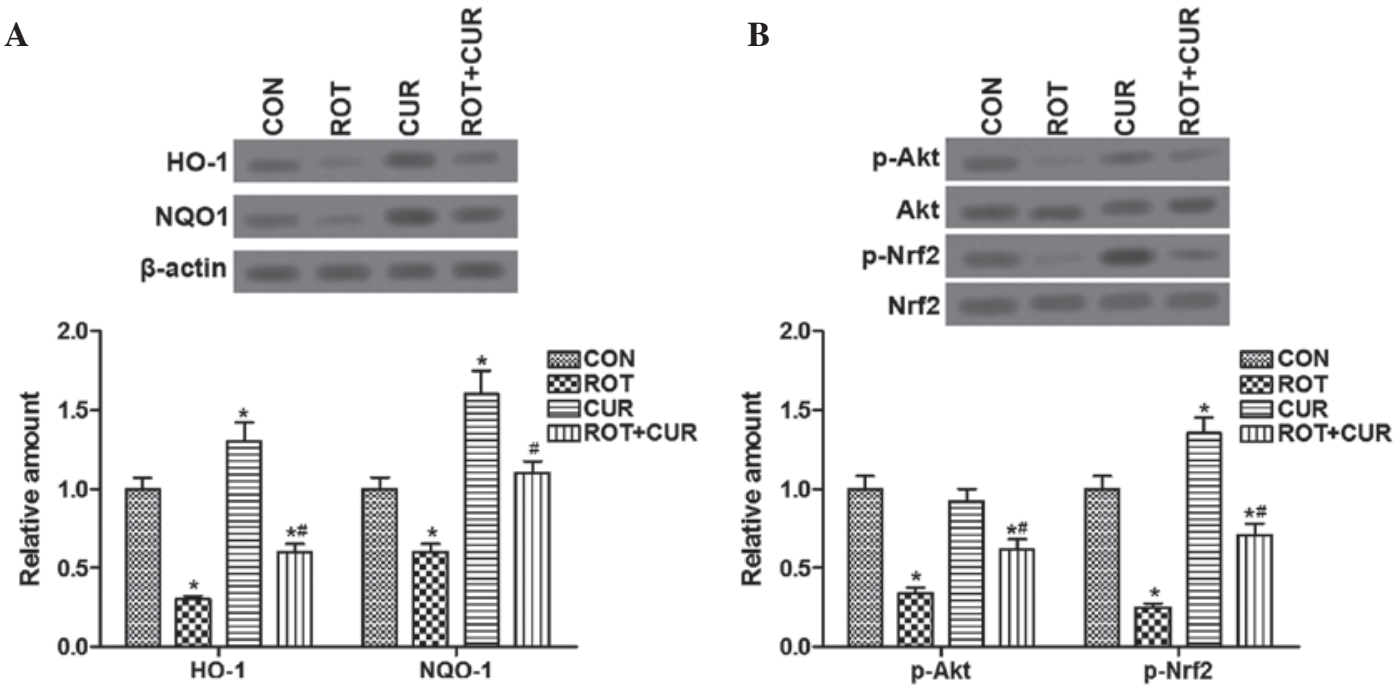

Figure 3. Effects of curcumin (CUR) and rotenone (ROT) on the Akt/nuclear factor erythroid 2-related factor 2 (Nrf2) signaling axis in the substantia nigra pars compacta of rats. The protein expression levels of (A) heme-oxygenase (HO)-1, NAD(P)H:quinone oxidoreductase 1 (NQO1) and $\beta$-actin, and (B) Nrf2, phosphorylated (p)-Nrf2, Akt and p-Akt were detected by western blotting. Quantification analysis was performed using Gel-Pro Analyzer 4.0 and normalized to $\beta$-actin in (A) and normalized to total Akt or total Nrf2 in (B). Data are presented as the mean \pm standard deviation. ${ }^{*} \mathrm{P}<0.05$ vs. the CON group. ${ }^{~} \mathrm{P}<0.05$ vs. the ROT group $(\mathrm{n}=6)$.

significantly reduced in cells treated with shNrf2 lentivirus or LY294002 (Fig. 4C). These results indicate that the neuroprotective effects of curcumin against ROT-induced SNpc dopaminergic neuronal oxidative damage may be inhibited by treatment with a shNrf2 lentivirus or LY294002.

\section{Discussion}

PD is regarded as a neurodegenerative disease, which is characterized by the age-associated accumulation of oxidative damage in human brain tissue. ROT, which is a commonly used 
A
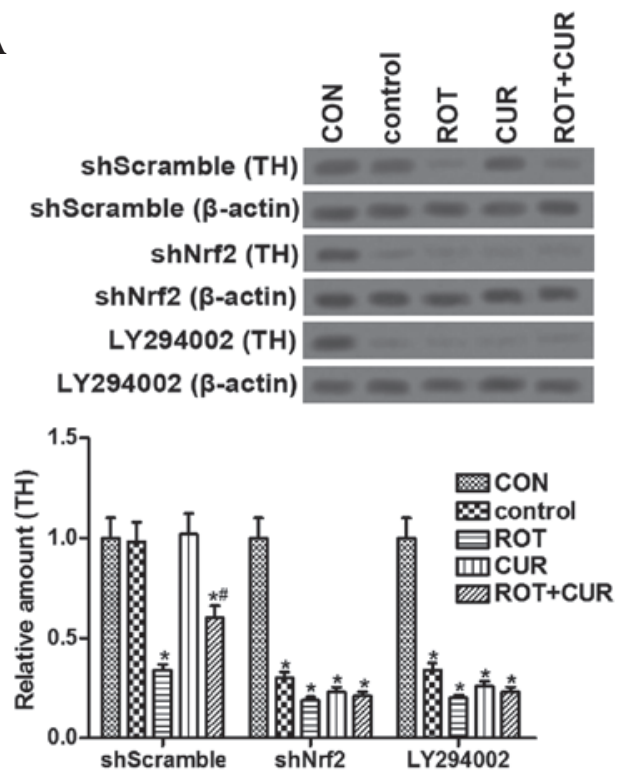

B
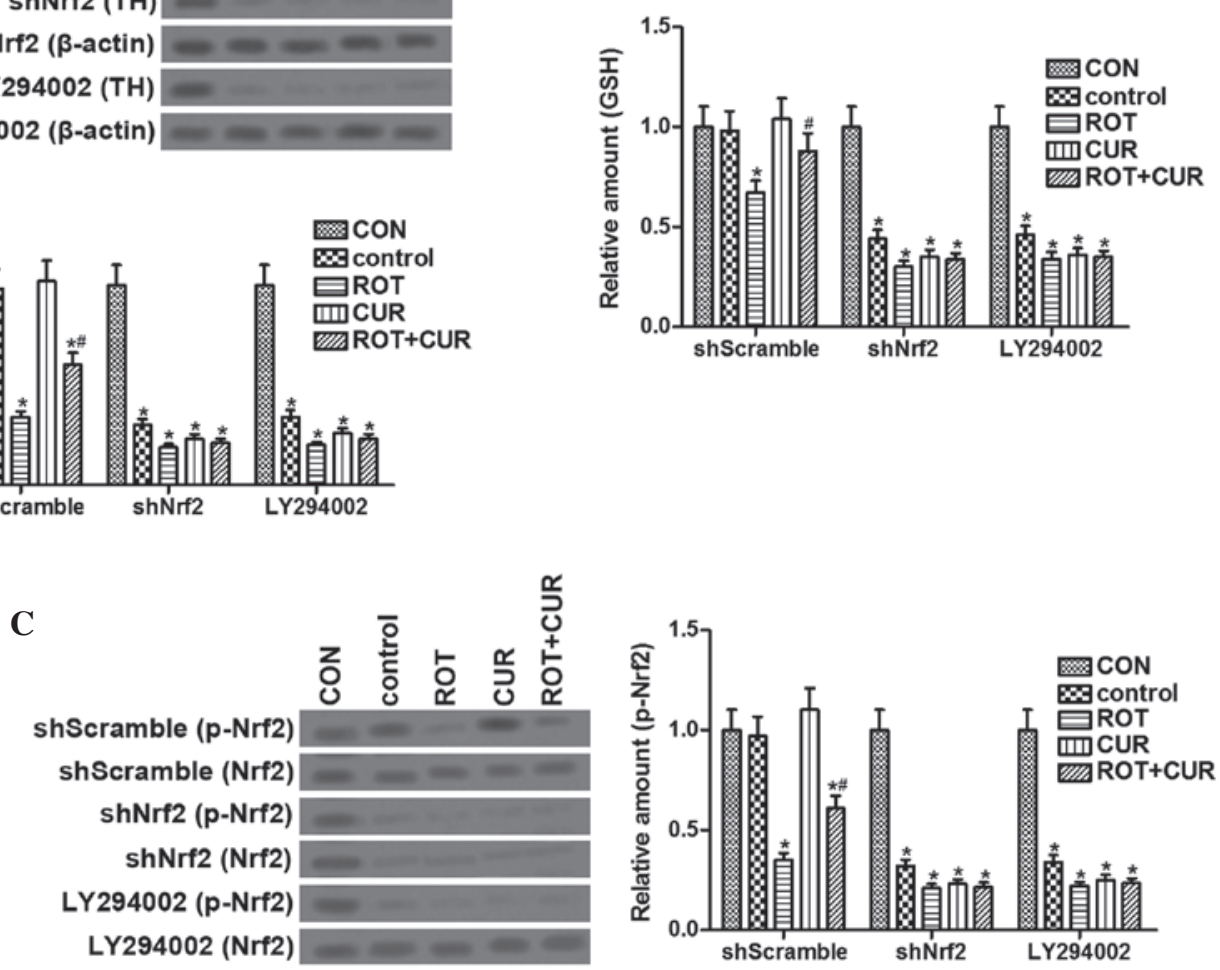

Figure 4. Effects of short hairpin RNA nuclear factor erythroid 2-related factor 2 (shNrf2) lentivirus and LY294002 treatment on the neuroprotective effects of curcumin (CUR) on rotenone (ROT)-induced substantia nigra pars compacta dopaminergic neuronal oxidative damage. (A) Protein expression levels of tyrosine hydroxylase (TH) and $\beta$-actin, as determined by western blotting. (B) Glutathione (GSH) levels were examined using a commercial kit. (C) Protein expression levels of phosphorylated (p)-Nrf2 and Nrf2, as detected by western blotting. Quantification analysis of western blotting was performed using Gel-Pro Analyzer 4.0 and normalized to $\beta$-actin in (A) and total Nrf2 in (C). CON group was untreated. Control group was treated with shScramble, shNrf2, or LY294002. Data are presented as the mean \pm standard deviation. ${ }^{*} \mathrm{P}<0.05$ vs. the CON group. ${ }^{\prime \prime} \mathrm{P}<0.05$ vs. the ROT group (n=6). Control group was regarded as the group treated with shScramble, shNrf2, or LY294002.

natural substance obtained from the roots of certain tropical plants including Derris elliptica, is capable of causing mitochondrial perturbation and oxidative damage (27). ROT is a lipophilic compound that can freely cross cell membranes and gain access to cytoplasm and mitochondria (28). Curcumin, which is a major active polyphenol component of turmeric, exerts various biological effects, and has been reported to possess antioxidative activity against oxidative damage in cell and animal models of PD $(15,17)$. The aim of the present study was to examine whether, and how, curcumin exerted protective effects against ROT-induced dopaminergic neuronal oxidative damage in a rat model of PD.

To better understand the therapeutic effects of drugs specific to certain disease processes, animal models are often used. Rats have been reported to develop motor and postural deficits characteristic of PD following ROT minipump administration for 35 days (29). Consistent with previous research, the present study successfully established a ROT-based rat model of PD, the chronic exposure of which was administered by subcutaneous injection. In addition, the effects of ROT could be significantly ameliorated with curcumin pretreatment. The majority of studies regarding ROT have concentrated on the neurotoxicity and behavioral deficits of neurodegenerative diseases, such as PD (8,30-32). For instance, ROT has been shown to result in motor impairment (32), behavioral deficits (31) and loss of TH immunoreactivity in the striatum or $\mathrm{SNpc}$ (8). Furthermore, curcumin has previously exhibited potential therapeutic protection by alleviating neurotoxic behavioral changes in mice (22), and notably exhibited a protective role in a 6-hydroxydopamine rat model of $\mathrm{PD}$, where it suppressed the degeneration of nigral dopaminergic neurons (9). In the present study, parkinsonian symptoms were assessed using various behavioral tests, including rotational behavior, catalepsy and open field tests; the results indicated that curcumin pretreatment was able to partly alleviate PD symptoms in the rats, and upregulate the expression levels of $\mathrm{TH}$, which were disordered by ROT.

A previous study demonstrated that the levels of endogenous oxidative markers (ROS and MDA), redox status (GSH), and response of antioxidant enzymes were associated with the state of oxidative stress in rat brain tissues (33). ROT has been shown to induce oxidative stress by stimulating the production of ROS (34) and MDA (35), and by reducing GSH levels in vitro and in vivo $(30,36)$. In addition, curcumin is 
able to cross the blood-brain barrier and has been reported to exert neuroprotective effects in neurological disorders (10), which could enhance the release of GSH (17), and decrease the levels of MDA (17) and ROS (18), in order to protect against oxidative damage in vitro and in vivo (16). The results of the present study demonstrated that curcumin was able to reverse ROT-induced effects, resulting in an elevation in GSH generation, and a reduction in the levels of ROS and MDA.

It is well documented that Nrf2 is a basic leucine zipper transcription factor that protects various tissues and cells from ROS via the antioxidant response element (ARE)-mediated induction of diverse antioxidant proteins and phase II detoxifying enzymes, including HO-1 and NQO1 $(21,37)$. GSH depletion results in oxidative stress and $\mathrm{TH}$ activity loss, which are considered symbolic events in neurodegeneration, including in the progression of PD (17,34). Curcumin may protect cortical neurons and reduce oxidative stress levels, and it has been hypothesized that these neuroprotective effects are regulated by the Akt/Nrf2 antioxidant system $(38,39)$. In addition, curcumin has been shown to affect the gene expression of phase II enzymes (40), inducing the antioxidant defense mechanism by activating the Nrf2 pathway and upregulating the expression levels of Nrf2/ARE pathway-related genes HO-1 and NQO1 $(38,41)$. The present study indicated that the beneficial effects of curcumin were due to changes in antioxidant enzyme activities, including $\mathrm{HO}-1$ and NQO1, via the activation of PI3K/Akt and transcription of Nrf2. In addition, continuous infusion with shNrf2 lentivirus or pharmacological inhibition with LY294002 suppressed Nrf2 phosphorylation and suppressed the expression levels of $\mathrm{TH}$ and GSH in the SNpc, indicating that the Nrf2-associated cytoprotective and antioxidative effects of curcumin are dependent on the PI3K/Akt pathway.

In conclusion, the present study established a rat model of PD using ROT, and examined the behavioral performance, endogenous markers of oxidative stress, the activities of antioxidant enzymes, and the activation of the related signaling pathways in the SNpc following curcumin pretreatment. The results indicated that the protective effects of curcumin against ROT-induced dopaminergic neuronal oxidative damage were mediated by activation of the Akt/Nrf2 signaling pathway. These results demonstrated that curcumin exerts potential neuroprotective therapeutic effects, and may be considered a novel therapeutic agent for the treatment of PD.

\section{References}

1. Nutt JG and Wooten GF: Clinical practice. Diagnosis and initial management of Parkinson's disease. N Engl J Med 353: 1021-1027, 2005.

2. Camilleri A and Vassallo N: The centrality of mitochondria in the pathogenesis and treatment of parkinson's disease. CNS Neurosci Ther 20: 591-602, 2014.

3. Mariani E, Polidori MC, Cherubini A and Mecocci P: Oxidative stress in brain aging, neurodegenerative and vascular diseases: An overview. J Chromatogr B Analyt Technol Biomed Life Sci 827: 65-75, 2005

4. Tolleson CM and Fang JY: Advances in the mechanisms of Parkinson's disease. Discov Med 15: 61-66, 2013.

5. Sayre LM, Smith MA and Perry G: Chemistry and biochemistry of oxidative stress in neurodegenerative disease. Curr Med Chem 8: 721-738, 2001.

6. Perry TL and Yong VW: Idiopathic Parkinson's disease, progressive supranuclear palsy and glutathione metabolism in the substantia nigra of patients. Neurosci Lett 67: 269-274, 1986.
7. Bharath S, Hsu M, Kaur D, Rajagopalan S and Andersen JK: Glutathione, iron and Parkinson's disease. Biochem Pharmacol 64: 1037-1048, 2002.

8. Saravanan KS, Sindhu KM, Senthilkumar KS and Mohanakumar KP: L-deprenyl protects against rotenone-induced, oxidative stress-mediated dopaminergic neurodegeneration in rats. Neurochem Int 49: 28-40, 2006.

9. Du XX, Xu HM, Jiang H, Song N, Wang J and Xie JX: Curcumin protects nigral dopaminergic neurons by iron-chelation in the 6-hydroxydopamine rat model of Parkinson's disease. Neurosci Bull 28: 253-258, 2012.

10. Mythri RB and Bharath MM: Curcumin: A potential neuroprotective agent in Parkinson's disease. Curr Pharm Des 18: 91-99, 2012.

11. Dutta S, Padhye S, Priyadarsini KI and Newton C: Antioxidant and antiproliferative activity of curcumin semicarbazone. Bioorg Med Chem Lett 15: 2738-2744, 2005.

12. Biswas SK, McClure D, Jimenez LA, Megson IL and Rahman I: Curcumin induces glutathione biosynthesis and inhibits NF-kappaB activation and interleukin- 8 release in alveolar epithelial cells: Mechanism of free radical scavenging activity. Antioxid Redox Signal 7: 32-41, 2005.

13. Singh $\mathrm{S}$ and Khar A: Biological effects of curcumin and its role in cancer chemoprevention and therapy. Anticancer Agents Med Chem 6: 259-270, 2006.

14. Daniel S, Limson JL, Dairam A, Watkins GM and Daya S: Through metal binding, curcumin protects against lead- and cadmium-induced lipid peroxidation in rat brain homogenates and against lead-induced tissue damage in rat brain. J Inorg Biochem 98: 266-275, 2004.

15. Ataie A, Sabetkasaei M, Haghparast A, Moghaddam AH and Kazeminejad B: Neuroprotective effects of the polyphenolic antioxidant agent, Curcumin, against homocysteine-induced cognitive impairment and oxidative stress in the rat. Pharmacol Biochem Behav 96: 378-385, 2010.

16. Jagatha B, Mythri RB, Vali S and Bharath MM: Curcumin treatment alleviates the effects of glutathione depletion in vitro and in vivo: Therapeutic implications for Parkinson's disease explained via in silico studies. Free Radic Biol Med 44: 907-917, 2008.

17. Harish G, Venkateshappa C, Mythri RB, Dubey SK, Mishra K, Singh N, Vali S and Bharath MM: Bioconjugates of curcumin display improved protection against glutathione depletion mediated oxidative stress in a dopaminergic neuronal cell line: Implications for Parkinson's disease. Bioorg Med Chem 18: 2631-2638, 2010.

18. Chen J, Tang XQ, Zhi JL, Cui Y, Yu HM, Tang EH, Sun SN, Feng JQ and Chen PX: Curcumin protects PC12 cells against 1-methyl-4-phenylpyridinium ion-induced apoptosis by bcl-2-mitochondria-ROS-iNOS pathway. Apoptosis 11: 943-953, 2006.

19. Cui QLSS: Curcumin antagonizes rotenone-induced injury of PC12 Cells by antioxidant activity. Acta Med Univ Sci Technol Huazhong 39: 37-46, 2010.

20. Tsou YH, Shih CT, Ching CH, Huang JY, Jen CJ, Yu L, Kuo YM, Wu FS and Chuang JI: Treadmill exercise activates Nrf2 antioxidant system to protect the nigrostriatal dopaminergic neurons from MPP+ toxicity. Exp Neurol 263: 50-62, 2015.

21. Lee JM and Johnson JA: An important role of Nrf2-ARE pathway in the cellular defense mechanism. J Biochem Mol Biol 37: 139-143, 2004.

22. Kakkar V and Kaur IP: Evaluating potential of curcumin loaded solid lipid nanoparticles in aluminium induced behavioural, biochemical and histopathological alterations in mice brain. Food Chem Toxicol 49: 2906-2913, 2011.

23. Mokrasch LC and Teschke EJ: Glutathione content of cultured cells and rodent brain regions: A specific fluorometric assay. Anal Biochem 140: 506-509, 1984

24. Shinomol GK and Muralidhara: Prophylactic neuroprotective property of Centella asiatica against 3-nitropropionic acid induced oxidative stress and mitochondrial dysfunctions in brain regions of prepubertal mice. Neurotoxicology 29: 948-957, 2008.

25. Ohkawa H, Ohishi $\mathrm{N}$ and Yagi K: Assay for lipid peroxides in animal tissues by thiobarbituric acid reaction. Anal Biochem 95: 351-358, 1979.

26. Lim JH, Kim KM, Kim SW, Hwang O and Choi HJ: Bromocriptine activates NQO1 via Nrf2-PI3K/Akt signaling: Novel cytoprotective mechanism against oxidative damage. Pharmacol Res 57: 325-331, 2008.

27. Lin TK, Cheng CH, Chen SD, Liou CW, Huang CR and Chuang YC: Mitochondrial dysfunction and oxidative stress promote apoptotic cell death in the striatum via cytochrome $c /$ caspase-3 signaling cascade following chronic rotenone intoxication in rats. Int J Mol Sci 13: 8722-8739, 2012. 
28. Coulom $\mathrm{H}$ and Birman S: Chronic exposure to rotenone models sporadic Parkinson's disease in Drosophila melanogaster. J Neurosci 24: 10993-10998, 2004.

29. Betarbet R, Sherer TB, MacKenzie G, Garcia-Osuna M, Panov AV and Greenamyre JT: Chronic systemic pesticide exposure reproduces features of Parkinson's disease. Nat Neurosci 3: 1301-1306, 2000.

30. Madathil SK, Karuppagounder SS and Mohanakumar KP: Sodium salicylate protects against rotenone-induced parkinsonism in rats. Synapse 67: 502-514, 2013.

31. Santiago RM, Barbieiro J, Lima MM, Dombrowski PA, Andreatini R and Vital MA: Depressive-like behaviors alterations induced by intranigral MPTP, 6-OHDA, LPS and rotenone models of Parkinson's disease are predominantly associated with serotonin and dopamine. Prog Neuropsychopharmacol Biol Psychiatry 34: 1104-1114, 2010.

32. Swarnkar S, Singh S, Mathur R, Patro IK and Nath C: A study to correlate rotenone induced biochemical changes and cerebral damage in brain areas with neuromuscular coordination in rats. Toxicology 272: 17-22, 2010.

33. Shinomol GK and Muralidhara: Bacopa monnieri modulates endogenous cytoplasmic and mitochondrial oxidative markers in prepubertal mice brain. Phytomedicine 18: 317-326, 2011.

34. Madathil KS, Karuppagounder SS, Haobam R, Varghese M, Rajamma U and Mohanakumar KP: Nitric oxide synthase inhibitors protect against rotenone-induced, oxidative stress mediated parkinsonism in rats. Neurochem Int 62: 674-683, 2013.
35. Liu CB, Wang R, Pan HB, Ding QF and Lu FB: Effect of lycopene on oxidative stress and behavioral deficits in rotenone induced model of Parkinson's disease. Zhongguo Ying Yong Sheng Li Xue Za Zhi 29: 380-384, 2013 (In Chinese).

36. Zhang Q, Zhang J, Jiang C, Qin J, Ke K and Ding F: Involvement of ERK1/2 pathway in neuroprotective effects of pyrroloquinoline quinine against rotenone-induced SH-SY5Y cell injury. Neuroscience 270: 183-191, 2014

37. Nguyen T, Sherratt PJ and Pickett CB: Regulatory mechanisms controlling gene expression mediated by the antioxidant response element. Annu Rev Pharmacol Toxicol 43: 233-260, 2003.

38. Wu J, Li Q, Wang X, Yu S, Li L, Wu X, Chen Y, Zhao J and Zhao Y: Neuroprotection by curcumin in ischemic brain injury involves the Akt/Nrf2 pathway. PLoS One 8: e59843, 2013.

39. Qin XY, Cheng Y and Yu LC: Potential protection of curcumin against intracellular amyloid beta-induced toxicity in cultured rat prefrontal cortical neurons. Neurosci Lett 480: 21-24, 2010.

40. Dickinson DA, Iles KE, Zhang H, Blank V and Forman HJ: Curcumin alters EpRE and AP-1 binding complexes and elevates glutamate-cysteine ligase gene expression. FASEB J 17: 473-475, 2003.

41. Gao S, Duan X, Wang X, Dong D, Liu D, Li X, Sun G and Li B: Curcumin attenuates arsenic-induced hepatic injuries and oxidative stress in experimental mice through activation of Nrf2 pathway, promotion of arsenic methylation and urinary excretion. Food Chem Toxicol 59: 739-747, 2013. 